\title{
Enhancing the accuracy of respiratory diagnoses in primary care: a report on the establishment of a Community Respiratory Assessment Unit
}

\author{
Rosemarie Hassett $^{a}$, Karen Meade ${ }^{a}$, Martyn R. Partridge ${ }^{b, *}$
}

\author{
${ }^{a}$ Hammersmith \& Fulham Primary Care Trust, London, UK \\ ${ }^{b}$ Department of Respiratory Medicine, Imperial College, \\ NHLI Division at Charing Cross Hospital Campus, St Dunstan's Road, London W6 8RP, UK
}

Received 3 August 2006; accepted 2 October 2006

\section{KEYWORDS}

Respiratory diseases;

Diagnosis;

Service provision;

Primary care:

Spirometry,

Community Respiratory

Assessment Unit

\section{Summary}

Aims: To establish a Commonil, Respiratory Assessment Unit and to evaluate its role in enhancing it e ccuracy of respiratory diacrosis in primary care.

Morion Ne established a sencra and beripatetic nurse-led service utilising semi-structured history takins, spirometry, oxygen saturation monitoring and semi-stractured eporting, coupled with the provision of educational materials to Lor primary care physicians and patients.

Results: Phased access to the service was offered to 32 general practices. Use varied widely between practices and a total of 364 patients were referred in the first year. The single biggest diagnostic group consisted of patients with definite or suspected COPD, but the diagnosis was often not confirmed. Patient and GP satisfaction with the service was extremely high; without it misdiagnoses and inappropriate therapeutic trials are possible.

Conclusion: A community respiratory assessment unit such as this is one way of offering a centrally-directed, quality-controlled, diagnostic support service for primary care physicians.

(C) 2006 General Practice Airways Group. Published by Elsevier Ltd. All rights reserved.

\section{Introduction}

The death rates from respiratory disease in the UK are amongst the highest in Europe and currently

\footnotetext{
* Corresponding author. Tel.: +44 208846 7587; fax: +44 2088467999.

E-mail address: m.partridge@imperial.ac.uk (M.R. Partridge).
}

lung disease kills one in four people in the UK [1]. Respiratory disease is also the most common illness responsible for an emergency medical admission to hospital and is the most common reason for patients to visit their general practitioner (GP); almost a third will visit their GP at least once a year because of a respiratory condition [1]. Such a burden of disease necessitates assurance that best practice is being implemented, and within the 
UK there are extremely good evidence-based guidelines for most common respiratory conditions produced by the British Thoracic Society (www. brit-thoracic.org.uk). Such guidelines can only be used appropriately if a correct diagnosis has been made and it needs to be recognised that the common symptoms of cough and breathlessness are shared with disorders of other systems. Breathlessness may thus reflect lung disease, heart disease, pulmonary vascular disease, diaphragm weakness, or systemic disorders such as anaemia or obesity, and cough may reflect lung disease, sinus disease, gastro-oesophageal reflux or use of medication such as ACE inhibitors.

Spirometry is one tool which might enhance diagnostic accuracy and we have previously shown that without use of spirometry, mistaken diagnoses are likely in primary care [2]. Various reports have been published of the appropriateness, usefulness and accuracy of spirometry performed in primary care [3], and of different ways of providing such a service [4]. However, because of concerns regarding the accuracy and usefulness of spirometry performed in primary care, and some GPs' reluctance to use the technique, one eminent author recently suggested that "perhaps the focus should shift for a few years from trying to convince primary care physicians to perform spirometry in their office to providing high awality espipatoly care services in each communi ty enthich primary

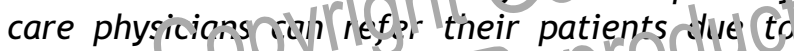
dyspnoea av COPD. These services could include convenient smoking cessation programmes, asthma education, spirometry, allergen skin testing, exhaled nitric oxide measurements. . . all following evidence-based clinical practice guidelines" [5].

Hospitalisation rates for the common respiratory diseases of asthma and chronic obstructive pulmonary disease (COPD) within Hammersmith \& Fulham Primary Care Trust (PCT) are amongst the highest in London [6]. In 2004 the Hammersmith \& Fulham PCT, with the support of the North West London Strategic Health Authority, Hammersmith Hospitals NHS Trust, and Imperial College London, set up a Community Respiratory Assessment Unit (CRAU) which aimed to:

- Improve the diagnosis of respiratory conditions in the community

- Empower patients with respiratory disease

- Encourage the implementation of the recommendations of national guidelines on the management of asthma, COPD, and other lung diseases.
This report describes the establishment and results of the first year's operation of the Hammersmith \& Fulham CRAU.

\section{Methods}

To develop and run CRAU, Hammersmith \& Fulham PCT recruited a specialist nurse with experience in respiratory medicine; this post was matched by a further nurse with the same experience employed on a fixed term contract by the Strategic Health Authority with the aim of ensuring that there were sufficient personnel to both run and evaluate the service. The service was developed by these two key personnel in consultation with GP colleagues and with the assistance of a local respiratory consultant (MRP). Both nursing members of staff underwent further respiratory training at the National Respiratory Training Centre (now called Education for Health) and underwent specific training in the use of spirometry. During the development period considerable attention was paid to the logistics of referral of patients to tite service to the siting of the service and the development of a protocol base f ap inch to patients being referred G the CRAU. A semi-standardised reporting system was idditent This report system was dosizn fic to leed back to GPs the relevant aspects of their patient's history, the results of spirometry and (where appropriate) bronchodilator testing, carbon monoxide monitoring, estimation of arterial oxygen saturation and information regarding inhaler techniques. In addition, a series of educational materials suitable for a variety of respiratory scenarios were developed which were sent back with the CRAU reports to the GPs. The aim was that the information would be of value to GPs for use in other patients. Such materials included reminders as to the causes of breathlessness, the causes of small lung disorders, the causes of unexplained cough, one-page summaries of the COPD and asthma guidelines, explanations regarding the so called Royal College of Physicians Three Questions designed to detect asthma morbidity [7] and aids to the differentiation of asthma from COPD $[7,8]$. Further material on smoking cessation and use of nicotine replacement therapy was available.

At the start of this project there were 33 general practices in the Hammersmith \& Fulham PCT. To avoid overwhelming the service and to permit easier evaluation of the benefits, 16 practices were given access to the service initially and a further 17 practices six months later (one general 


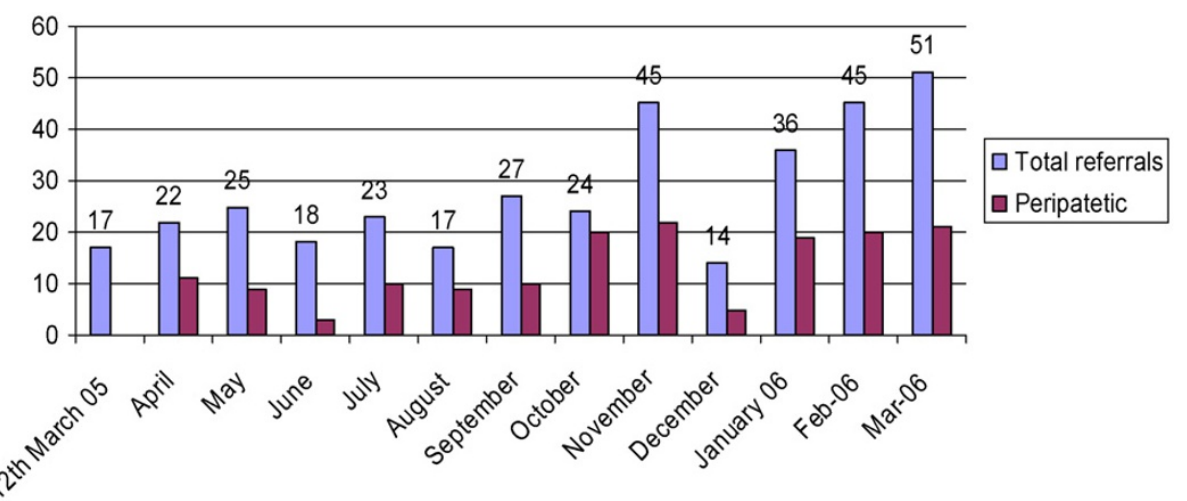

Figure 1 Total number of referrals per month (with those seen in the peripatetic service shown separately).

practice subsequently closed). The specialist nurses visited each of these practices to inform them of the service, and the Medical Chairman of the Professional Executive Committee of the PCT also wrote to each GP about the service. Referral forms were added to local GPs' computers where possible. Although a community service, the base for the centre was initially within Charing Cross Hospital adjacent to a primary/secondary care respiratory nursing service but a peripatetic service was also offered to practices at the northernmost and southernmost parts of the PCT, geographicaly farthest away from Charing Cross Hospitat

As part of the referral proces ops were asked to state on the referial form whether the diagnos:s was definie or suspected astiriv (ar COPD, or they were given the option of stating that the problem was one of an unexplained cough or unexplained breathlessness. The request form also required GPs to state what they would have done if the Community Respiratory Assessment Unit had not been available. Request forms were faxed to CRAU and the patients were given an information leaflet by their GP and asked to telephone for an appointment at a mutually convenient time. For the peripatetic service, dates for visits were agreed with the practices, who then contacted the patients and the GP completed the referral form.

\section{Results}

Referrals

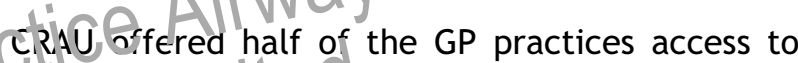
the service frorn enalch 2005 and the remaining pratiles haciaccess from September 2005. A total of 364 patients were referred in the first 12 months, for whom we have complete data for 330; 140 in the first six months and 190 in the second six month period. 148 of the referrals were male $(45 \%)$ and 182 were females (55\%). The age ranged from 18 to 90 years (mean 62.97, SD 14.9). 36 percent were smokers $(107 / 299)$ and $41 \%$ ex-smokers $(123 / 299)$.

The total number of referrals per month of operation of the service is shown in Figure 1.

Practices 1-16 access for 12 months

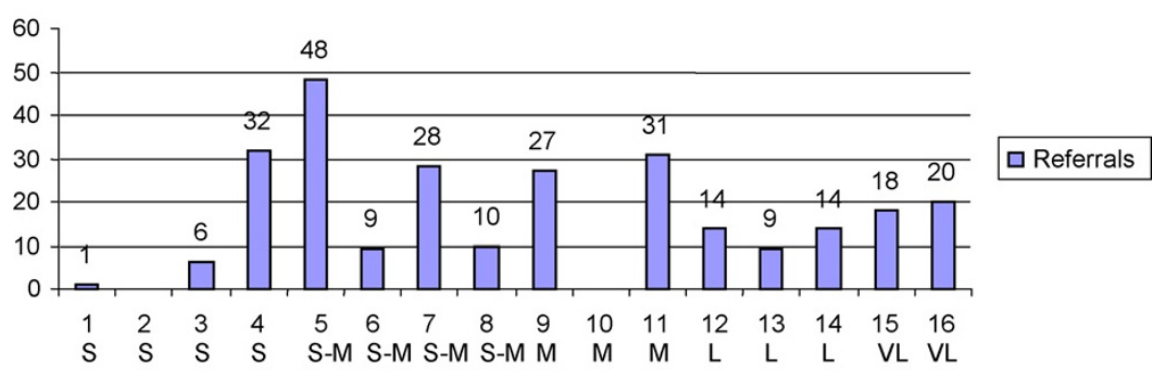

The Initials under the Practice Identification codes reflect the practice size;

$\mathrm{S}($ Small $)=$ Under 3000 patients, S-M (Small to Medium $)=3001-5000, \mathrm{M}$ (Medium)

$=5001-7000, \mathrm{~L}($ Large $)=7001-10000$ and $\mathrm{VL}($ Very Large $)=10001-13000$.

Figure 2 Referrals from practices 1-16 who had access to CRAU for the whole 12-month period. 
Practices $17-33$ access for 6 months only

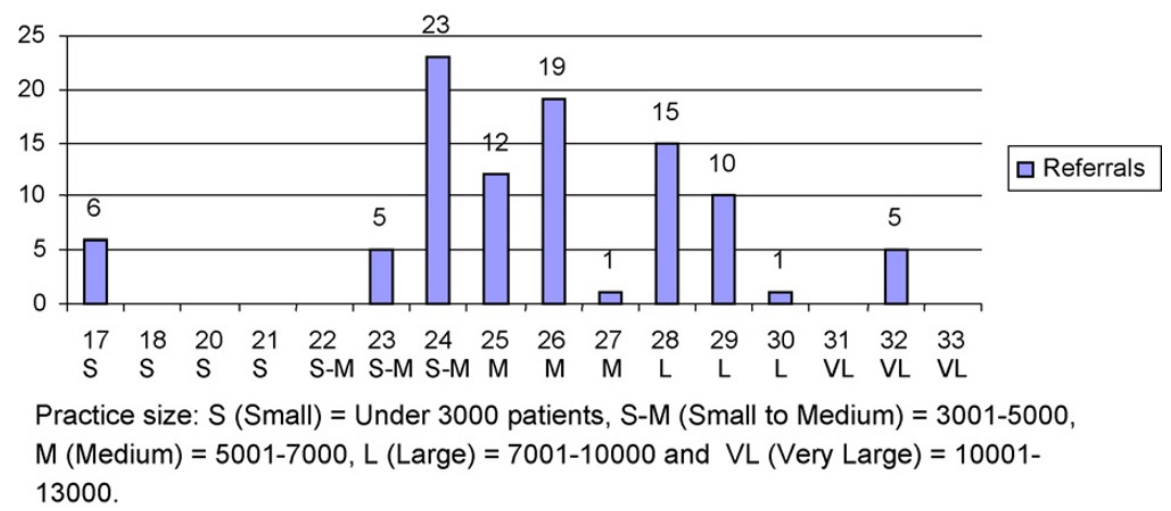

Figure 3 Referrals for those practices who had access for 6 months only.

The peripatetic service practices were chosen for geographical reasons, but not all GP practices offered this service wished to take part, usually due to difficulties with space. In the first phase, seven out of eight practices accepted the peripatetic service, and in the second phase four of the eight practices accepted the offer.

There was significant variation in referral rates from different practices, as shown in Figures 2 and 3.

Figure 3 shows the number of referrals from leach practice that had access to CRAQ (1) y or the last six-month period. In the tirit priase, two practices out of 16 madono referrals and in the ceconid phase, six practices made no reisr als. Referrals amongst the other practices varied between 1 and 48 referrals per month.

The reasons for referral are shown in Figure 4, from which it can be seen that the single largest diagnostic category is that of "suspected COPD" with "suspected" or "definite" COPD together accounting for 189 referrals (57\%).
The outcome in terms of diagnoses suggested by CRAU on their report to the referring GP is shown in Figure 5. From this, it can be seen that whilst definite or suspected COPD was the most common reason for referral $(189 / 330-57 \%$ of all referrals), airway narrowing was only demonstrated in 110 of those 189 cases (58\%). Eight of those patients

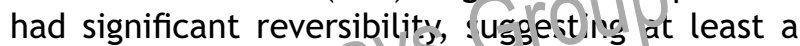
significant compone ts of asthma. A quarter of all Dat er ts ifferred with definite or suspected COPD chad no abnormalities at all detected during the assescrient Defirite or suspected asthma account 10. $2 \%$ of all referrals $(92 / 330)$, and in $34 \%$ of those $(31 / 91)$ airway narrowing was demonstrated. In ten of those cases $(32 \%)$, there was definite significant confirmed reversibility.

One hundred and twelve patients were referred with suspected or stated COPD or asthma, or both, but shown to have no airway narrowing on spirometry. 19 of these patients $(17 \%)$ had an unexpected restrictive/small lung disorder, and $53 \%$ of those $(10 / 19)$ had a BMl greater than

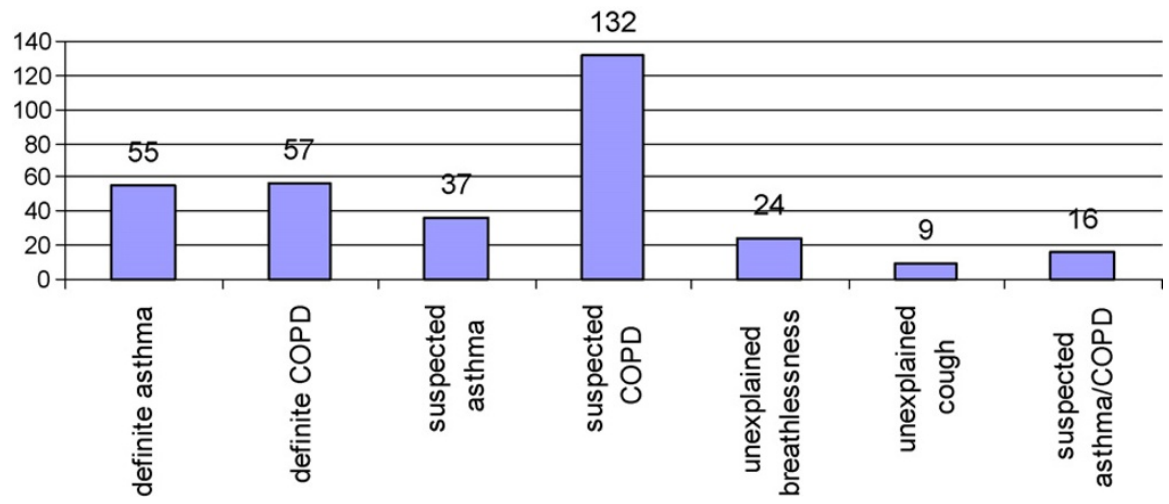

Figure 4 Reason for referral. 


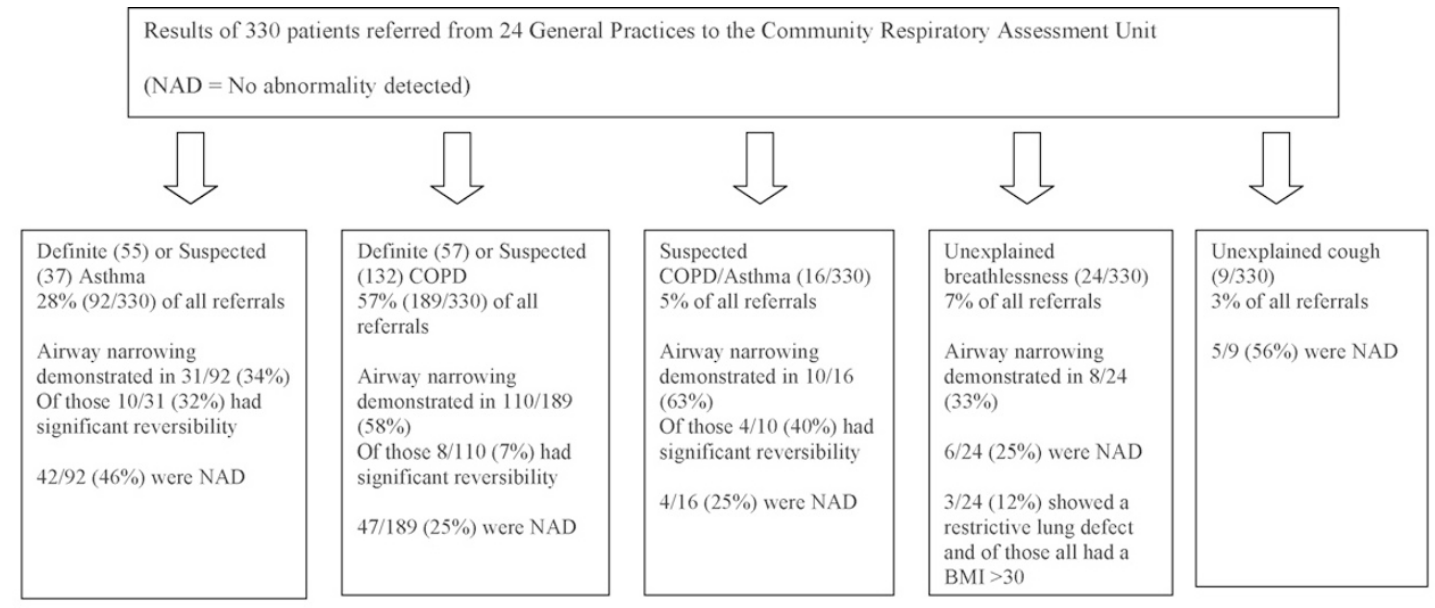

\begin{tabular}{|ll|}
\hline - Technically unsatisfactory & $8 / 330(2 \%)$ \\
- Did not attend appointment & $21 / 330(6 \%)$ \\
- Unable to proceed & $4 / 330(1 \%)$ \\
- Combined obstructive/restrictive pattern & $2 / 330(.6 \%)$ \\
- Other & $10 / 330(3 \%)$ \\
\hline
\end{tabular}

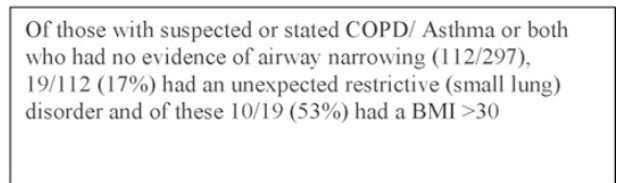

Figure 5 Results of patients referred to CRAU.

30. The BMI of the 298 patients referred for whom we have data was as follows: $\mathrm{BMI}<25=14 \%$; $25-29.9=27 \%$; $>30=29 \%$.
- $25 \%(9 / 20)$ vely helpful - $\forall 2 \%(26 / 36)$ helpf

- 3\% (1/36) at)hepful

iwo GPs commented that they had not seen any educational information.

$97 \%(38 / 39)$ were happy with the length of time from referral to receipt of report and 3\% (1/39) were not.

Of those GPs who had been receiving the peripatetic service:

- $62 \%(8 / 13)$ were satisfied

- $38 \%(5 / 13)$ were very satisfied with the service

Those GPs who did not have the peripatetic service were asked if they would have liked access to this service and $8 / 9(89 \%)$ replied positively. Overall satisfaction with the service was extremely high: $46 \%(18 / 39)$ were very satisfied; $51 \%(20 / 39)$ were satisfied; and one GP (3\%) was neither satisfied nor dissatisfied. $88 \%$ of GP respondents (35/40) felt that their patients had benefited or greatly benefited from the service.

\section{Action which would have been taken if CRAU was not available}

As part of the referral process, the GPs were asked to state how they would have managed the patients if the CRAU had not been available. 


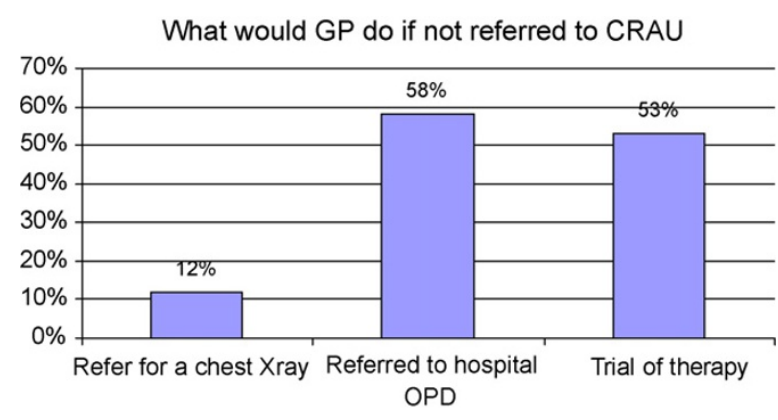

Figure 6 What would the GP do if CRAU unavailable.

Figure 6 demonstrates the results and shows that $58 \%$ of cases would, according to the GP, have been referred to a specialist hospital respiratory outpatient clinic if the CRAU did not exist, and $53 \%$ would have had a trial of therapy. (Clearly, the GPs could tick multiple alternative options for each patient for this section.)

In the $58 \%$ of cases where the GP said that they would refer the patient to a hospital outpatient clinic, a third stated a "respiratory clinic", 28\% specifically mentioned Charing Cross Hospital, and $18 \%$ the Hammersmith Hospital. 21 percent stated "other hospitals" or did not specify. We used the information regarding suggested referral to the Hammersmith, Charing Cross or "a respiratorv clinic" and tried to assess the impast of this is ated behaviour against actual iefe ra! to to respiratory

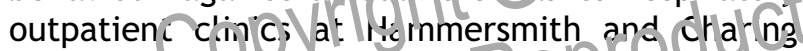

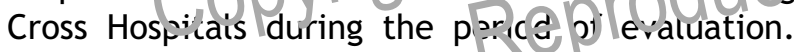
The mean number of referrals to Hammersmith Hospital NHS Trust (Charing Cross and Hammersmith Hospitals) for the year 2004/2005 was $29.75 \pm 5.62$ per month from this PCT, and the mean number of referrals for year 2005/2006 was 29.91 \pm 8.55 . Figure 7 plots the number of cases per month which
GPs said they would have sent to a respiratory clinic locally had CRAU not been available, against the actual number of outpatient referrals received (from the PCT); the data for the "potential avoided referrals" has been deliberately delayed in this figure by four weeks, to allow for delay between referral and patients being seen in secondary care.

\section{Trials of therapy}

$53 \%$ of GP referrals said that if CRAU had not been available, they would give a trial of therapy. $70 \%(98 / 140)$ mentioned a prescription for a short-acting beta-agonist, $53 \%(74 / 140)$ an inhaled corticosteroid, 31\% (44/140) a long-acting beta-agonist, $6 \%(9 / 140)$ tiotropium and $3 \%$ (4/140) antibiotics. Of those patients who were referred with suspected or stated COPD in whom spirometry turned out to be normal, in 24 out of 81 cases the GP would, without the service, have provided the patient with a prescription; in ten cases for a short-acting beta-agonist, in eight an inhaled corticosteroid, in three cases a long-acting beta-agonist, and in two cases an anti-cholinergic age 14 . Imt $(N)$ C C ases the GP would have given antibistics

\section{Patisik Satisfaction (2)uestionnaire results}

To a:Sess d d dient satisfaction with the service they received at the CRAU, each patient who attended was sent a questionnaire and a stamped addressed envelope for its return. Not all patients responded to all of the questions; for example those attending the peripatetic service did not respond to questions regarding ease of access to Charing Cross Hospital. 291 questionnaires were sent to patients, and 208 (71\%) were returned.

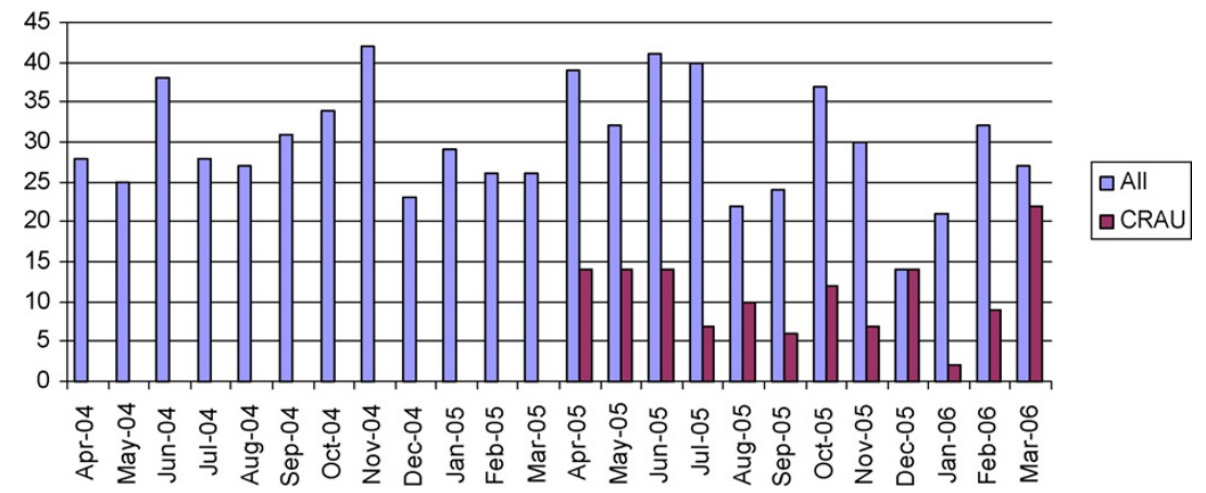

Figure 7 Average number of referrals per month to a Hammersmith Hospitals NHS Trust respiratory clinic (at either Charing Cross of Hammersmith Hospital) for GPs employed by Hammersmith and Fulham PCT NHS Trust. The number of patients referred to CRAU whom the GP stated they would have otherwise referred to a specialist clinic is also shown. 
Overall satisfaction with the service was extremely high:

- $99 \%(196 / 198)$ of all patients who attended CRAU for respiratory assessment following referral from their GP rated the experience as good $(29 / 196)$, very good $(66 / 196)$, or excellent (101/196).

\section{CRAU service design results showed that:}

- $95 \%(192 / 203)$ of patients knew why they were referred to CRAU.

- $92 \%(179 / 194)$ found it easy to obtain an appointment at CRAU.

- The patient information leaflet was found to be informative or very informative by $97 \%(163 / 168)$ of the patients.

- The appointment time was convenient in $98 \%$ $(196 / 200)$ of cases.

- $97 \%$ felt that the length of the consultation with the specialist nurse was satisfactory; $1 \%(2 / 194)$ felt that the appointment time was too long, and $2 \%$ felt that the appointment length of 45 minutes was not long enough.

In response to questions regarding their acquisition of knowledge from CRAU:

- $63 \%(113 / 179)$ of patients stated that thev had learnt something new about thein inhaler treatment at CRAU.

- $94 \%(196 / 197)$ fel Chat the verbal informatio recei ed during the consuliation '́as scevit.

- $63 \%(67 / 107)$ felt that the written information they received during the consultation was useful.

\section{Discussion}

This report describes the establishment and use of a Community Respiratory Assessment Unit (CRAU) designed to enhance the accuracy of respiratory diagnoses and the implementation of optimal care in the general practices of one London PCT. A number of important messages emerge from the establishment of this Unit and from evaluation of its first year of operation.

COPD represents the major reason for referral of patients to this service and the results suggest that if such referral had not taken place, mistaken diagnoses would have occurred in a third of cases and there was a potential for significant inappropriate medications to be given to patients in whom the diagnosis was not confirmed by spirometric demonstration of airway narrowing. As in a previous study [2], referral for spirometry was not shown to be so helpful for those with suspected or stated asthma, and this often reflected referral of patients who were well at the time of referral or referral of those who had already been started on anti-inflammatory therapy. Under such circumstances it is impossible to tell whether the normality of the results reflected benefit of the treatment or the absence of a diagnosis of asthma. Special educational materials were produced for primary care physicians to emphasise the symptoms which suggested asthma, the differentiation of asthma from COPD, and the need wherever possible to confirm objectively a diagnosis of asthma by demonstrating variable airway narrowing. It was stressed that while spirometry was one way of doing this (pre- and post-bronchodilator), supply of a peak flow meter for the patient to undertake domiciliary peak flow monitoring, or inducing airway narrowing where appropriate by an exercise test, were suitable alternatives.

Within the group of patients being referred with unexplained breathlessness, and also in the group being referred with other stated or suspected diagnoses who were found to have unexpected restrictive defects, were many patiel's who had a $\mathrm{BMI}$ in the obese or overiveiot t range. We formed the impresion wat CPs were not always aware Git the rikelihood of lexcess weight as a cause of breathlessness. $\mathrm{e} 0$

(11) is likely that some of the initial pattern of referral of patients to this service, and possibly the dominance of COPD amongst those referred, reflects the UK Government's Quality Outcome Framework for GPs \{Department of Health QOF, $2004430 /$ id $\}$ whereby there is a financial reward for confirming a diagnosis of COPD by spirometry. However, the service was never designed to be merely a spirometry service, albeit a centrally-directed, quality-controlled service, and materials were produced for GPs which they were encouraged to use for their wider population of patients. GPs' satisfaction with the service, its organisation, its educational materials and its value to patients was extremely high, as was patient satisfaction.

Studies amongst those patients with asthma have suggested a preference for services to be provided as near to patients' homes as possible [9] and we consider that this service could eventually be totally peripatetic. However, provision of a peripatetic service was not always as popular as we had envisaged, and this reflected the fact that many GPs did not have space available for a visiting service. A peripatetic service could only be available on a limited time basis, and some GPs commented that they preferred the ready 
availability of an 'any day of the week' central service.

Whether the CRAU service has had a value other than that demonstrated by usage or reported satisfaction is harder to verify. Clearly, GPs reported that without the service they would sometime have initiated therapies subsequently shown to be potentially inappropriate, and some hospital referrals were probably avoided. The effect of enhanced implementation of guidelines and the potential effect of the 'drip feed' of educational materials has to be seen within the context of multiple simultaneous influences. We do have baseline prescribing data for most of the practices with access to the service and would anticipate revisiting the impact of the service on harder outcomes including prescribing and hospital referral rates in due course. Such evaluation will include comparison between users of the service and those not referring patients. For others considering setting up a similar service we believe that the current demand could be met using one 'whole time equivalent' respiratory nurse specialist. This person, or two part-timers, could work most effectively as part of a community or 'interface' respiratory nursing team.

\section{References}

[1] British Thoracic Society. The burden of lung disease. A statistical report from the British Thoracic Society. $\mathrm{Br}$ Thoracic Society 2000.

[2] Wolfenden H, Bailey L, Murphy K, Partridge MR. Use of an open access spirometry service by general practitioners. Prim Care Resp J 2006;15(4):252-5.

[3] Lusuardi M, De Benedetto F, Paggiaro P, Sanguinetti C, Brazzola G, Ferri $P$, et al. A randomized controlled trial on office spirometry in asthma and COPD in standard general practice: data from spirometry in Asthma and COPD: a comparative evaluation Italian study. Chest 2006;129:844-52.

[4] Jones R, Whittaker M, Hanney K, Shackell B. A pilot study of a mobile spirometry service in primary care. Prim Care Resp J 2005;14:169-71.

[5] Enright P. Does screening for COPD by primary care physicians have the potential to cause more harm than good? Chest 2006;129:833-5.

[6] Saxena S, George J, Barber, Fitzpatrick J, Majeed A. Association of population and practice factors with potentially avoidable admission rates for chronic diseases in London: cross sectional analysis. J Royal Soc Med 2006;99:81-9.

[7] British Thoracic Society. Scottish Intercollegiate Guidelines Network: British Guideline on the Management of Asthma. Thorax 2003; 58:i1-i94.

[8] NICE. Chronic obstructive pulmonarv disease - ivanagement of chronic obstructive pulmonary discase ir ad to in primary and secondary care. Thin ar 201)4;5\%.s1-232.

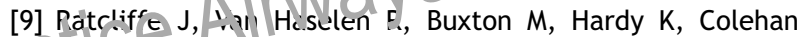
J Dartidge MR. Asselising patients preferences for characteristics assoiat with homeopathic and convenciora treatment of asthma: a conjoint analysis. Tr $\mathrm{c}=\mathrm{a}$ 2002;57:503-8.

\section{Conflict of interest}

There are no conflicto (it in erest co declare
Available online at www.sciencedirect.com

ScienceDirect 